\title{
Introduction to the Special Collection of Communications from ELSI 2014
}

\author{
Henderson James Cleaves ${ }^{1,2,3}$
}

Received: 17 December 2014 / Accepted: 20 December 2014 /

Published online: 11 July 2015

(C) Springer Science+Business Media Dordrecht 2015

Keywords Origin of life $\cdot$ Hadean $\cdot$ Early evolution $\cdot$ Planetary science

This special section presents contributed short papers from the 2nd Annual Earth-Life Science Institute International Symposium, which was convened at the National Institute of Informatics in Chiyoda-ku, Tokyo, from March 24th to 26th, 2014. The meeting was attended by about 200 scientists and featured 34 invited speakers from seven countries. Presentations were divided between five oral presentation sessions covering the Origin \& Evolution of the Earth-Life System, including 1. Origin of Life: Scenarios \& Approach, 2. Water in the Early Solar System, 3. Where Did Life Emerge? Deep Sea, Surface, or Mars?, 4. Exploring the Hadean Earth and 5. Early Evolution of Earth and Life before Oxygen, as well as a poster flash talk session and 3 poster sessions. More information, including a complete speaker list can be found at: http://www.elsi.jp/en/research/activities/symposium/2014/03/000922.html.

Henderson James Cleaves henderson.cleaves@gmail.com

1 Earth-Life Science Institute, Tokyo Institute of Technology, Tokyo, Japan

2 Institute for Advanced Study, Princeton, NJ, USA

3 Blue Marble Space Institute for Science, Washington, DC, USA 